\title{
Depth of comprehension, effective elaboration, and memory for sentences
}

\author{
ROSEMARY J. STEVENSON \\ University of Durham, Durham DHI 3LE, England
}

\begin{abstract}
It was hypothesized that depth of comprehension is a function of the complexity of the meaning components of a sentence that are activated during initial encoding. In four experiments, subjects were presented sentences containing either transitive causative verbs (e.g., "John opened the door") or their intransitive noncausative counterparts (e.g., "The door opened") and were required to produce a continuation to each sentence. Based on the above hypothesis, sentences for which the continuations induced causative interpretations should be remembered better than sentences for which noncausitive interpretations were induced. The results confirmed this hypothesis, and they are considered in relation to depth of comprehension, effective elaboration, and the role of inferences in memory for sentences.
\end{abstract}

Work from a number of different contexts converges on the view that depth of comprehension is an important factor in memory for words and sentences. It has been proposed, for example, that the more elements of the meaning of a word that are activated during encoding of a word list, the greater is the likelihood that the word will be recalled (Johnson-Laird, Gibbs, \& de Mowbray, 1978) and that sentences that require elaborative contributions for their comprehension (e.g., "The shirt looked terrible because Jane ironed it") are more likely to be recalled than are sentences that do not require such cognitive elaborations (e.g., "The shirt looked terrible so Jane ironed it") (Bransford \& McCarrell, 1974). The unifying feature of these two approaches is that they both imply that depth of comprehension is related to effective elaboration (see, e.g., Stein, Morris, \& Bransford, 1978).

This notion of effective elaboration has come about mainly in reaction to the view that deep semantic processing is activated in an all-or-none fashion (e.g., Hyde \& Jenkins, 1969; Walsh \& Jenkins, 1973) and as a result of more recent work that suggests that there are different degrees of semantic processing (e.g., Craik \& Tulving, 1975; Stein et al., 1978). Craik and Tulving (1975), for example, found that subjects recalled more words requiring a "yes" answer to the encoding question than words requiring a "no" answer, and this led Craik and Tulving to suggest that elaboration of encoding was

Most of this article is based on a doctoral dissertation submitted to the University of London and supported by the SSRC. Portions of this article were presented at the meeting of the Experimental Psychology Society, London, 1977. I would like to thank Tim Shallice, my dessertation supervisor, for his helpful suggestions and guidance, Phil Johnson-Laird for his useful and constructive comments on the original dissertation, Fergus Craik for his helpful advice on an earlier version of this paper, David Kleinman for attempting to clarify my prose, and John Boyle for help with the rating procedures used in Experiment 3. as important as depth of encoding. However, the theoretical underpinnings of both depth of comprehension and effective elaboration are frequently hard to specify. The notions are often invoked on the strength of the investigator's intuition, which may not be shared by others (see, e.g., Postman \& Kruesi, 1977) and are sometimes inferred from performance at recall, which only results in circularity (Nelson, 1977).

One way to provide an a priori specification of depth or elaboration, and hence to increase precision when discussing degrees of semantic processing, is to relate it to a well-defined psycholinguistic analysis (JohnsonLaird et al., 1978; Marslen-Wilson \& Tyler, 1976). Thus, Johnson-Laird et al. (1978) refer to amount of processing, which they define in terms of the number of meaning elements of a word that are activated during encoding. An approach like this provides an independent criterion for what the subject is actually doing during comprehension. However, since it involves certain theoretical assumptions, it also raises the possibility of a more detailed interpretation of previous studies on, for example, effective elaboration. Thus, while Stein and Bransford (1979) and Stein et al. (1978) have elucidated the constraints on effective elaboration, their interpretation can be extended. Stein and Bransford (1979) found that the target word "fat" was more likely to be remembered when it was embedded in a sentence such as "The fat man read the sign warning about thin ice" than when it was embedded in a sentence such as "The fat man read the sign that was two feet high." Stein and Bransford concluded that effective elaboration specifies the potential significance of facts rather than simply making the facts more memorable.

But what exactly is involved when the subject recognizes the potential significance of the word "fat" in the first sentence that is not involved in the comprehension of the second sentence? One possibility is that in order to comprehend the first sentence the implications, or 
meaning elements, of the relative adjective "fat" need to be explicitly considered, whereas in the second sentence these implications are unnecessary: The semantic facts associated with the adjective do not need to be activated in order for the second sentence to be understood. This analysis suggests that the main function of effective elaboration during encoding is to allow the activation of a more complex semantic structure of the target item, a view that follows from a definition of amount of processing in terms of the activation of meaning elements associated with the target item. The present studies, therefore, are an attempt to test this view by defining depth of comprehension in terms of the number of meaning elements that are activated during encoding. A second aim is to extend the work of Johnson-Laird et al. (1978) by using sentences rather than words as the items to be recalled.

The sentences that were used contained either transitive, causative verbs or their intransitive, noncausative counterparts (e.g., "open"). Despite the controversy among linguists over the precise nature of the underlying representation of causative constructions (see, e.g., Shibitani, 1976), it is usually assumed that causative sentences (e.g., "John opened the door") have more complex underlying structures than do noncausative sentences (e.g., "The door opened"). Thus Fillmore (1968) proposes that these verbs have a single lexical entry whose case frame specifies that an object noun is obligatory, whereas agent and instrumental nouns are optional. If only the object noun is selected, the verb functions as an intransitive, noncausative verb; but if the agent and/or the instrumental nouns are chosen, the verb functions as a transitive, causative verb. Hence the causative form involves at least one extra case role. While workers in generative (e.g., McCawley, 1972) and interpretive (e.g., Katz, 1972) semantics disagree over whether or not the causative form is derived transformationally from the intransitive, noncausative form, they do agree that the semantic structure of the causative form requires an extra clause that contains the verb of causing. So here, too, the causative form has a more complex structure. Stemming from a somewhat different tradition is the work of Miller and Johnson-Laird (1976) on procedural semantics. They propose that verbs like "open" contain three arguments in their underlying, procedural representations. When the transitive, causative form is encountered, all three of these arguments are evaluated; but when the corresponding noncausative form is encountered, only the first of these three arguments is evaluated (see Miller \& Johnson-Laird, 1976, p. 544). Hence, once again, the causative form is more complex than the corresponding noncausative. Clearly, there is no unanimous agreement on the precise formulation of causative constructions, but whatever the preferred formulation, there is general agreement that causative sentences have a more complex semantic representation than do their noncausative counterparts. In the present study, therefore, if the experimental sentences receive a causative interpretation, we will assume that they have been more deeply comprehended than if they recieve a noncausative interpretation. This follows from a definition of depth of comprehension in terms of the complexity of the underlying structure that is processed. We would expect, therefore, that sentences that receive a causative interpretation should be remembered better than sentences that receive a noncausative interpretation. This follows from the view that the more deeply a sentence is comprehended, the more likely it is to be remembered.

In Experiment 1, the subjects produced continuations for sentences containing either transitive or intransitive verbs. The transitive verbs occurred in truncated passive sentences (e.g., "The door was opened"), and it was assumed that continuations to these sentences would contain the missing actors. For the intransitive sentences (e.g., "The door opened"), it was assumed that the most natural continuation would be some kind of adverbial phrase (e.g., "The door opened very suddenly"). Thus the transitive sentences should be interpreted causatively and the intransitive sentences should be interpreted noncausatively, and according to a depth-ofcomprehension view, the transitive sentences should subsequently be recalled better than the intransitive sentences. In Experiment 2, only the intransitive sentences were used, and the subjects were instructed to produce causal continuations and hence should have interpreted these intransitive sentences causatively. Again, based on a depth-of-comprehension view, we would expect these subjects to recall the presented sentences better than do subjects who needed only to interpret the sentences in their noncausative sense in order to produce continuations. Experiment 3 used only transitive sentences and examined whether the superior recall of these sentences in Experiment 1 was genuinely due to their causative interpretation. The subjects were instructed to produce particular kinds of continuations, which either allowed the causative component to be activated or rendered it unnecessary. Again, we would expect that the sentences would be remembered best when the causative component was activated. The causative interpretations of the sentences in Experiments 1,2, and 3 were all induced by continuations that invariably involved an animate actor, and it is possible that it was this feature that accounted for the superior recall of sentences receiving this interpretation (see, e.g., Clark \& Begun, 1971). Experiment 4 investigated this possibility with transitive sentences by instructing one group of subjects to produce continuations that contained an instrument rather than an actor. If it is the notion of an animate actor that enhances recall, then subjects who produce actor continuations should recall the presented sentences better than do subjects who produce instrument continuations. If, however, it is the causative interpretation that enhances recall, then both groups of subjects should recall the sentences equally well and better than subjects whose continuations did not require a causative interpretation of the sentences. 


\section{EXPERIMENT 1}

This experiment tests the depth-of-comprehension hypothesis by using a sentence-completion task and comparing recall of the presented sentences that contained either transitive causative or intransitive noncausative verbs. The transitive sentences were truncated passives, and it was expected that the continuations were most likely to contain the missing actors. The intransitive sentences, however, were vague as to the presence of an agent. Kintsch (1972) found that 6 of 27 subjects produced an actor for the sentence "The cake bakes" when he asked them to write down what else they know about particular sentences. Thus it seems plausible that subjects can interpret the intransitive sentences causatively, but that it is not very likely they will do so. It seems more natural to expect the continuations to these sentences to contain adverbial phrases, which do not require a causative interpretation of the sentences. If the transitive and intransitive sentences do receive causative and noncausative interpretations, respectively, then according to a depthof-comprehension view, the transitive sentences should be recalled better than the intransitive sentences in a subsequent recall task.

\section{Method}

Subjects. Twenty undergraduate and postgraduate volunteers from University College London served as subjects and were paid for their participation. The ages ranged from 18 to 32 years. Each subject was tested individually.

Materials. A pool of 80 sentences was produced by pairing each of 20 transitive/intransitive verbs with two low-frequency nouns ( 9 or less in Thorndike \& Lorge, 1944). Low-frequency nouns were used to ensure that the sentences were sufficiently unusual to preclude guessing. Each noun was used twice in compiling the sentences, once in a transitive sentence (the noun was verbed) and once in an intransitive sentence (the noun verbed). Randomized sets of these 80 sentences were presented to 30 independent subjects, who were asked to rate them on a 7-point scale for frequency of occurrence in spoken English. The sentences that were used in the experiment were selected from the rare end of this scale, again as a control against guessing.

Two lists of 14 sentences were prepared from this sample. The sentences in the two lists contained the same nouns and verbs and differed only in whether the auxiliary "was" was present (transitive list) or absent (intransitive list). The 14 verbs are listed in the Appendix.

Procedure. Ten subjects were assigned to each condition. One condition consisted of the transitive sentences, and the other condition consisted of the intransitive sentences. The subjects were told that the experimenter was interested in how well people understood the meanings of sentences. The 14 sentences in each condition were presented orally, and the subjects were required to write down a sensible continuation to each sentence that continued the sense of the sentence. Subjects were urged to produce a continuation for every sentence and were further instructed that they must not use a particular continuation more than once. The sentences were presented in a different random order to each subject. On completion of this task, the subjects handed their continuations to the experimenter and were then asked to take part in another, unrelated experiment (an instructed sentence memory experiment, in which the sentences were all declaratives, e.g., "John listened to the news on the radio"). When the second experiment was completed, the subjects received an unexpected memory task for the transitive or intransitive sentences and were asked to write down as many of the presented sentences as they could remember. Subjects were allowed $10 \mathrm{sec}$ to write a continuation to each sentence. Approximately $15 \mathrm{~min}$ elapsed between completion of the continuations and recall of the presented sentences. A $3-\min$ period was allowed for recall of the sentences.

\section{Results and Discussion}

A sentence was scored as correct if it meant the same as the original sentence. Thus, synonymous words were accepted in place of the nouns and verbs: For example, for the test sentence "The malady was healed," the sentence "The malady was cured" was scored as correct. Similarly, for the test sentence "The increment enlarged," the sentence "The increment increased" was scored as correct. Whenever it made sense to do so (as in the last example), a synonym accepted in one condition was always accepted in the other condition. Whenever there was any doubt about the acceptability of a synonym, a second judge, who did not know the purpose of the experiment, was consulted, and the second judge's decision was used for that sentence.

The mean number of transitive sentences correctly recalled was 5.1 , and the mean number of intransitive sentences correctly recalled was 3.3. Following Clark (1973), two analyses of variance were carried out on the data, one treating subjects as a random factor $\left(F_{1}\right)$ and one treating sentences as a random factor $\left(\mathrm{F}_{2}\right)$, and the values for $\min \mathrm{F}^{\prime}$ were computed. Use of two separate analyses means that there is no joint error mean square when min $F^{\prime}$ values are computed.

Because the sentences in the two conditions contained the same nouns and verbs, a repeated-measures analysis was used to compute $F_{2}$. The transitive sentences were recalled better than the intransitive sentences according to both the $F_{1}$ and $F_{2}$ analyses $\left[\mathrm{F}_{1}(1,18)=6.48, \mathrm{p}<.05, \mathrm{MSe}=2.5 ; \mathrm{F}_{2}(1,13)=6.43\right.$, $\mathrm{p}<.05, \mathrm{MSe}=4.09]$, and the min $\mathrm{F}^{\prime}$ value was marginal $\left[\min F^{\prime}(1,30)=3.23, p<.10\right]$. It was also suggested that actor continuations were most likely to be produced to the transitive sentences and that such continuations entailed a causative interpretation of the sentences. Intransitive sentences, on the other hand, were predicted to receive predominantly adverbial continuations, which did not entail a causative interpretation of the sentences. The type of continuations that were produced in the two conditions are shown in Table 1. This table also shows the probability of a sentence's being recalled, given the type of continuation produced. Since both actor and adverb phrase continuations were produced to the transitive sentences, it was possible to compare the recall of these sentences according to which of these two types of continuation had been produced. Using repeated-measures analyses of variance, it was found 
Table 1

Continutations Produced for Transitive and Intransitive Sentences and the Probability of a Sentence's Being Recalled Given That Continuation

\begin{tabular}{llllll} 
& \multicolumn{2}{c}{$\begin{array}{c}\text { Transitive } \\
\text { Sentences }\end{array}$} & & \multicolumn{2}{c}{$\begin{array}{c}\text { Intransitive } \\
\text { Sentences }\end{array}$} \\
\cline { 2 - 3 } \cline { 5 - 6 } & $\mathrm{N}$ & $\mathrm{P}$ & & $\mathrm{N}$ & $\mathrm{P}$ \\
\hline Actor & 41 & .59 & 0 & \\
Adverb Phrase & 33 & .27 & 81 & .26 \\
Cause & 20 & .45 & & .25 \\
Others & 36 & .17 & 43 & .25 \\
No Response & 10 & .30 & 12 & .08 \\
\hline
\end{tabular}

Note $-N=$ number produced $P=$ probability of recall.

that transitive sentences that had actor continuations had a greater probability of recall than did transitive sentences that had adverb phrase continuations $\left[F_{1}(1,9)=13.25, p<.01, M S e=.036 ; F_{2}(1,10)=13.34\right.$, $\left.\mathrm{p}<.01, \quad \mathrm{MSe}=.079 ; \quad \min \mathrm{F}^{\prime}(1,19)=6.65, \quad \mathrm{p}<.05\right]$ (three sentences had to be discarded from the $F_{2}$ analysis because no adverb phrase continuations were produced to them).

Thus it appears that recall of the transitive sentences, at least, did depend on the type of continuation that was produced. Production of actor continuations entails a causative interpretation of these sentences, but adverb phrase continuations do not require the sentences to be interpreted causatively. Hence, a causative interpretation seems to enhance the recall of the transitive sentences.

The predominant continuation to the intransitive sentences was an adverb phrase, $58 \%$ as opposed to $23 \%$ for the transitive sentences, which bears out the initial predictions and presumably accounts for the poorer recall of these sentences. The continuations to the transitive sentences were much more variable, however, and this could account for the min $F^{\prime}$ value failing to reach an acceptable level of significance when the overall recall of the two sets of sentences was compared. It is notable that the cause continuations to the transitive sentences also resulted in a fairly high probability of recall. Again, one would expect a cause continuation to require a causative interpretation of the sentences. The low probability associated with cause continuations to the intransitive sentences is probably due to the low number of such continuations in this condition. The predominant continuation in the "others" category of Table 1 was a description of some other event that could have occurred in conjunction with that described in the sentence (e.g., "The yacht was withdrawn and everybody cheered"). Again, this type of continuation does not require a causative interpretation of the sentence and the recall probabilities were correspondingly low.

However, a stricter test of the original hypothesis requires tighter control over the kinds of continuations that are produced, in order to show that continuations involving causative interpretations do lead to superior overall recall of the presented sentences. Furthermore, a better test would be to show that the same sentences are recalled differently, depending on the type of continuation that has been produced. Such tests were made in Experiments 2 and 3.

\section{EXPERIMENT 2}

In Experiment 1, the superior recall of transitive sentences with actor continuations over transitive sentences with adverb phrase continuations was attributed to a causative interpretation's being required for the former but not for the latter group of sentences. Similarly, the marginal superiority of recall of the transitive list compared with the intransitive list was attributed to the greater likelihood of the former sentences' receiving continuations that required a causative interpretation of the sentences (actors or causes). However, it might well be the case that transitive sentences are recalled better than intransitive sentences are for other reasons. The wider range of types of continuations, for example, might have provided more specific retrieval cues than was the case with the transitive sentences. In Experiment 2, therefore, only the intransitive sentences were used. Subjects were instructed to produce continuations to these intransitive sentences. The continuations differed in whether or not they entailed causative interpretations of the sentences. If it was the causative interpretation that produced the superior recall of the transitive sentences in Experiment 1, then it should be possible to improve the recall of intransitive sentences if subjects can be instructed to produce continuations that require a causative interpretation of them. Although an intransitive sentence like "The door opened" does not entail that someone (or something) must have caused the door to open, a person may well believe that this is, in fact, the case. Hence, instructing subjects to produce cause continuations should direct the subjects' attention to this possibility, and, on a depth-ofcomprehension hypothesis, such subjects should remember the sentences better than subjects not so instructed.

\section{Method}

Subjects. Thirty undergraduate and postgraduate volunteers from University College London served as subjects and were paid for their participation. Their ages ranged from 18 to 26 years. Each subject was tested individually.

Materials. The 14 intransitive sentences were the same as those used in Experiment 1.

Procedure. There were three experimental conditions, with 10 subjects assigned to each condition. The subjects in each condition were instructed to produce a particular type of continuation to the intransitive sentences. In the "cause" condition, the subjects were instructed to write continuations that contained a cause for each event described by the sentences. In the "description" condition, the subjects were instructed to provide a word or phrase to describe the event in each presented sentence. This corresponded to the predominant response in Experiment 1 . In the "concurrentevent" condition, the subjects were instructed that their continuations should describe another event that could have happened at the same time as the one 
described in the sentence but that did not follow from or bring about the event described in the sentence: it was to be totally unrelated. This condition was included in order to provide continuations of comparable syntactic complexity to those in the cause condition. At the end of the sentence-completion task, all subjects carried out an intervening digit task that lasted approximately $1 \mathrm{~min}$. In all other respects, the procedure was the same as that employed in Experiment 1.

\section{Results and Discussion}

The scoring procedure throughout these experiments was the same as that used in Experiment 1 . The mean numbers of intransitive sentences recalled in each condition were as follows: cause condition, 5.4 ; description condition, 2.9 ; concurrent-event condition, 3.2 .

An independent-groups analysis was used to compute $F_{1}$, and a repeated-measures analysis was used to compute $F_{2}$ (this was because the presented sentences were the same in the three conditions). These analyses indicated that there was a significant main effect of type of continuation $\left[\mathrm{F}_{1}(2,27)=10.59, \mathrm{p}<.01, \mathrm{MSe}=1.76\right.$; $\mathrm{F}_{2}(2,26)=6.83, \mathrm{p}<.05, \mathrm{MSe}=1.95 ; \min \mathrm{F}^{\prime}(2,50)=$ $4.2, \mathrm{p}<.05]$. Observation of the mean recall scores suggests that this effect was due to the superior recall of the sentences that had cause continuations relative to the sentences that had description or concurrentevent continuations. Newman-Keuls analyses using either $F_{1}$ or $F_{2}$ variance estimates indicated that the mean of the causal condition was significantly larger than the means of the two other conditions $(\alpha=.05)$. There was no significant difference between the means of the description and concurrent-event continuations. These results indicate that the intransitive sentences were indeed recalled differently according to the type of continuation that was produced. After a cause was provided for the event, recall of the sentences was much better than after a description of the event or another event was produced. The findings support the view that memory is facilitated when subjects interpret the sentences causatively.

It seems unlikely that the results reflect either the time required to make a continuation or its complexity. The subjects in the concurrent-event condition evidenced the greatest difficulty in producing a continuation, and informal observation suggested that they spent more time thinking about their continuations than did the other subjects. Similarly, the concurrent-event group produced complex clauses for their continuations, just as the subjects in the cause condition did. It appears, therefore, that the same sentences will be recalled differently depending on whether or not subjects interpret the sentence causatively. Nevertheless, there remains the possibility that in Experiment 1, the transitive sentences were, in general, recalled better than the intransitive sentences because they were in some way easier for reasons other than that they frequently received a causative interpretation, as required by the continuations. This proposition was investigated in Experiment 3.

\section{EXPERIMENT 3}

\section{Method}

Subjects. Twenty 1st-year Durham University undergraduates served as subjects. Their ages ranged approximately from 18 to 26 years. The subjects were unpaid and were tested in two groups of 10 subjects, each group participating in one of the two conditions.

Materials. To increase generality and precision, a larger pool of 163 transitive sentences was constructed. These sentences contained 26 different verbs, each of which was paired with a varying number of nouns ( 49 or less on the Thorndike \& Lorge, 1944, word count). Randomized sets of these 163 sentences were rated on a 7-point scale by two independent groups of subjects (undergraduates at Brunel University) for frequency of occurrence in spoken English and for comprehensibility. The mean frequency and comprehension ratings were computed for each sentence, and the sentences that were used in the experiment were selected on the basis of these means. This was to ensure that the sentences were equated for both rated comprehensibility and rated frequency of occurrence. The mean comprehensibility rating of the sentences was $2.6(\mathrm{SD}=.48)$ (the higher the rating, the greater was the comprehensibility), and the mean frequency rating was $2.72(\mathrm{SD}=.56)$ (the higher the rating, the greater was the frequency). The 14 verbs are listed in the Appendix.

Procedure. All the subjects heard the same list of transitive sentences. There were two experimental conditions, with 10 subjects being assigned to each condition. One group of subjects was instructed to produce actors in their continuations (actor condition); the other group of subjects was instructed to make their continuations a description of the event described in the sentence (description condition). Apart from the group testing, the remaining procedure was identical to that employed in Experiment 2. Since it is assumed that both cause continuations and actor continuations require a causative interpretation of the presented sentences, the switch from cause continuations that were used in Experiment 2 is probably not crucial. Actor continuations were used here because they were the most predominant continuations to the transitive sentences in Experiment 1 and the aim of this experiment was to check that the actor continuations were indeed responsible for the superior recall of the transitive sentences in Experiment 1.

\section{Results and Discussion}

In the actor condition, the mean number of sentences correctly recalled was 5.9 ; in the description condition, the mean number recalled was 2.6. An independentgroups analysis was used to compute $F_{1}$; a repeatedmeasures analysis was used to compute $F_{2}$. Both analyses, and the subsequent min $F^{\prime}$, indicated that recall in the actor condition was significantly higher than in the description condition $\left[\mathrm{F}_{1}(1,18)=12.05, \mathrm{p}<.01\right.$, $\mathrm{MSe}=4.52 ; \quad \mathrm{F}_{2}(1,13)=14.61, \quad \mathrm{p}<.01, \quad \mathrm{MSe}=2.66$; $\left.\min \mathrm{F}^{\prime}(1,31)=6.6, \mathrm{p}<.05\right]$.

Once again, it is the nature of the continuation that determines how well the sentence will be recalled. It is possible, however, that continuations containing an agent are more imageable than are continuations containing a description. If subjects use imagery, then this could account for the differences in recall. To check this possibility, each sentence with all its continuations was presented to an independent group of subjects, who rated them on a 5-point scale for ease of imageability. The overall imagery ratings were then computed for the 
Table 2

Mean Imagery Ratings and Mean Recall for the Sentences and Their Continuations

\begin{tabular}{lcc}
\hline & \multicolumn{2}{c}{ Continuation } \\
\cline { 2 - 3 } & Actor & Description \\
\hline Mean Imagery Rating & 3.08 & 3.05 \\
Mean Recall & 4.20 & 1.85 \\
\hline
\end{tabular}

sentences plus continuations produced by the actor group and by the description group. These imagery ratings are shown in Table 2 alongside the mean recall in each condition. The mean recall scores in Table 2 are averaged over sentences $(n=14)$, not subjects $(n=10)$, and hence are different from those presented above. The overall imagery ratings were very similar in the two conditions despite the differences in recall. Hence, it seems unlikely that the recall differences were due to differences in the imagery level of the continuations. The size of the correlation between the imagery ratings and the recall scores supports this suggestion $(\rho=.02)$.

One confounding feature of all these experiments is that all the actor continuations and the majority of the cause continuations involve the notion of an animate actor. Inspection of the cause continuations in Experiment 2 , for example, indicated that only $24 \%$ of them stated or implied that an instrument rather than actor had brought about the event (e.g., "The labyrinth widened because it had been eaten away by a stream," as opposed to "The malady healed because she had applied the ointment"). It is possible that the concept of an animate actor plays a central role in cognitive organization and that this could account for the superior recall of sentences with actor or cause continuations. To test this possibility, Experiment 4 was conducted.

\section{EXPERIMENT 4}

If it is the concept of an animate actor that was responsible for the superior recall associated with actor and cause continuations in the previous experiments, then we would expect actor continuations to result in better recall of transitive sentences than do instrument continuations. If, on the other hand, the superior recall was due to the sentences' being interpreted causatively, then, based on a depth-of-comprehension hypothesis, we would expect both actor continuations and instrument continuations to produce better recall of the sentences than would description continuations.

\section{Method}

Subjects. Forty-five 1st-year Durham University undergraduates served as subjects. Their ages ranged approximately from 18 to 26 years. The subjects were unpaid and participated in three group sessions at the beginning of three laboratory classes.

Materials. The 14 transitive sentences were the same as those used in Experiment 3.

Procedure. There were three experimental conditions, with 15 subjects being assigned to each condition. The actor condi- tion and the description condition were the same as those used in Experiment 3. In the instrument condition, the subjects were instructed that their continuations were to contain an instrument that could have caused the event described in each presented sentence. They were cautioned that these instruments should be inanimate objects. Each group session consisted of a varying number of subjects from each condition. The instructions were presented orally for all three conditions, and subjects were then assigned to their conditions. The remaining procedure was the same as that of Experiment 3.

\section{Results and Discussion}

The mean number of sentences recalled was 5.87 in the actor condition, 4.6 in the instrument condition, and 2.33 in the description condition. The two analyses of variance indicated that there was a significant main effect of condition $\left[\mathrm{F}_{1}(2,42)=12.11, \mathrm{p}<.01, \mathrm{MSe}=3.97\right.$; $\mathrm{F}_{2}(2,26)=8.459, \mathrm{p}<.01, \mathrm{MSe}=6.09 ; \min \mathrm{F}^{\prime}(2,58)=$ $4.98, \mathrm{p}<.05]$. Newman-Keuls analyses using either $F_{1}$ or $F_{2}$ variance estimates indicated that the means of both the actor condition and the instrument condition were significantly larger than the mean of the description condition $(\alpha=.05)$. Although the mean of the actor condition was higher than that of the instrument condition, this comparison was not significant with either of the variance estimates.

Thus the results support the view that facilitation of sentence recall is due to the sentences' receiving a causative interpretation and not to the fact that the facilitating continuations in the previous experiments contained animate actors. It seems, then, that the nature of the continuation can have a crucial effect on how well the original sentences will be remembered, a finding that is in line with that of Stein and Bransford (1979). In the present studies, though, the target items were sentences rather than target adjectives embedded in sentences, and the constraints on effective elaboration were related more specifically to the underlying semantic representations that are activated during encoding.

\section{GENERAL DISCUSSION}

Taken together, what do these experiments indicate? First of all, it seems that the nature of the sentences may determine the ease with which particular continuations will be produced (Experiment 1). Second, the nature of the continuations appears to determine the recall of the presented sentences. If a continuation entails the activation of a complex underlying structure for the presented sentence, then recall of that sentence will be enhanced. Third, it does seem to be the causative component rather than the actor component that is responsible for facilitating recall (Experiment 4); instrument continuations facilitate recall in the same way as actor and cause continuations do. The data therefore support the view that there are degrees of semantic processing. They also extend the findings from memory for word lists (Johnson-Laird et al., 1978) and memory for words in sentences (Stein \& Bransford, 1979) to memory for sentences. 
More particularly, though, viewing depth of comprehension as a function of the complexity of the underlying meaning elements that are activated during encoding gives the notion of "depth" a more precise specification and enables clear-cut predictions to be tested. In the present experiments, if the full causative structure is activated, then recall is better than if the noncausative structure is activated. Such findings are compatible with Bransford and McCarrell's (1974) view that the greater the cognitive elaboration that is required for comprehension of a sentence, the better it will be remembered. They also implement Stein et al.'s (1978) suggestion that clarification of the conditions under which semantic processing may facilitate comprehension and memory requires an examination of how people make use of their general knowledge (or semantic knowledge) to effectively elaborate information. Based on a depth-of-comprehension view, the causal components of transitive causative sentences will only be activated if the continuation involves a causal element (Experiments 3 and 4). The data of Experiment 1 suggest that this may occur quite naturally in a sentence continuation task with truncated passive causatives, whereas Experiment 2 suggests that the subjects can interpret the intransitive forms causatively if the task instructions require them to do so. Furthermore, it seems to be the complexity of the underlying structure that is activated that determines the recall of the sentences. Specifying the conditions under which people will construct increasingly complex semantic components should prove to be a valuable aid to understanding the ways in which material may be learned more effectively.

The arguments presented here rely very heavily on the ability to specify the nature of semantic representations. But Fodor, Fodor, and Garrett (1975) have argued that semantic representations have no psychological reality. They argue that it is intuitively implausible to assume that psychological complexity mirrors semantic complexity. The present data, however, suggest that predictions based on a semantic model do successfully predict psychological performance, and, in particular, the more complex the semantic structure that is activated, the easier it is to recall the sentence. Of course, the more complex semantic representations may not be activated during comprehension (as in the concurrent-event and description conditions of Experiments 2,3, and 4), but then the sentences will be less well remembered. It seems that the activation of complex semantic structures is not a necessary requirement for shallow comprehension, but that it is necessary for increasing degrees of comprehension and, hence, memory.

There is, however, an alternative view of the data from the present experiments, a view that is less linguistically based than the one presented above. This is that the superior recall of sentences receiving causal interpretations is not due to the complexity of the semantic structure that has been activated, but to the complexity of the cognitive representation that has been constructed. That is, the causal interpretation may involve the implementation of inference rules that make explicit the implications of the causal relation. The latter view seems to place greater emphasis on the processes involved in constructing a representation of the sentence than on the structure of the representation itself. However, a distinction between the effects of underlying structure and the effects of the processes involved in constructing a representation is difficult to establish empirically. But the major distinction between these two interpretations lies in the reliance on semantic (linguistic) components on the one hand and on more general cognitive components on the other hand. The procedure of the present experiments does suggest an empirical test of the semantic interpretation that was presented above: that the causal continuations activated a causative semantic structure. If this is the case, then producing causal continuations to sentences containing verbs that do not entail a causative component (e.g., "John knew the answer") should not enhance the recall of the sentences relative to the same sentences receiving noncausal continuations. This is because there would be no underlying causative representation of the verb that could be constructed. If, on the other hand, the superior recall of the sentences receiving causal continuations is due to the construction of a more cognitively based representation in which the implications of the causal relation have been made explicit, then even sentences with no causal component should be recalled better when they receive causal continuations than when they receive noncausal continuations. Such a test would not determine whether it is the structure of the representation or the processes involved in constructing the representation that influence recall. But it would distinguish between a purely semantic interpretation and a more general cognitive interpretation. Whatever the final interpretation, however, a semantic analysis of the materials to be remembered would seem to be a prerequisite for a more precise specification of both depth of comprehension and effective elaboration.

One further issue that might be considered more explicitly in the light of present experiments is the role of inferences in memory for sentences. Since producing an inference requires adding some extra information to the presented sentences, it can be argued that requiring subjects to write a continuation to a sentence enabled them to write down the most obvious inference from that sentence, particularly given the short period of time that they had available for the task. Viewed in this light, the experiments reported here present a different perspective on work on inference. Most studies of inference are concerned with demonstrating that people can make inferences and that these inferences are indistinguishable in memory from material that was actually presented (e.g., Brewer, 1977; Johnson, Bransford, \& Solomon, 1973). Implicit in this work is the assumption that different kinds of inferences have identical memorial consequences (e.g., Harris, 1974). The type of inference that a subject might make is not assumed to make any differences to the resulting memory structure. 
Furthermore, the emphasis has been on memory for the inferences themselves, and the possibility that different inferences will have different effects on the memorability of the explicit information has not been considered.

Viewing the sentence continuation task as a means of allowing the subjects to write down an inference from each sentence leads us to modify some of these assumptions. Experiment 1 suggests that the structure of the sentence will determine how readily particular inferences are drawn. Brewer (1977) has shown that sentences differ in the ease with which they will elicit inferences, but in that study, the sentences were selected on an intuitive basis. A more plausible approach seems to be to attempt to specify the nature of possible inferences by considering the possible semantic representations of the sentences. The transitive and intransitive sentences that were used here did allow a more precise specification of whether a causal or an adverbial inference might be drawn. More important, though, are the findings that sentence continuations that involved the inference of a cause led to better recall of the explicit information than did sentence continuations that involved adverbial or other event inferences. Such observations must cast doubt on any theoretical account that assumes that different inferences have the same psychological consequences; this does not seem to be the case when recall of the explicit information is considered. The data also imply that increasing depths of comprehension are not simply a function of the number of inferences that are made from an assertion (Monaco \& Harris, 1978), but they are also dependent upon the nature of the inferences that are made. If an inference induces the activation of complex semantic (or cognitive) information associated with the asserted sentence, then the sentence is more likely to be remembered. Such an analysis has the merit of tying together the work on inferences with work on depth of comprehension and effective elaboration, and the unifying feature would seem to be a consideration of the semantic requirements of the task at hand. If the notion of depth of comprehension is to be rigorous enough for formal testing, then a semantic analysis of the sentences that are used would seem to be a useful analytical tool.

\section{REFERENCES}

Bransford, J. D., \& McCarrell, N. S. A sketch of a cognitive approach to comprehension: Some thoughts about understanding what it means to comprehend. In W. B. Weimer \& D. S. Palermo (Eds.), Cognition and the symbolic processes. Hillsdale, N.J: Erlbaum, 1974.

BREWER, W. F. Memory for the pragmatic implications of sentences. Memory \& Cognition, 1977, 5, 673-678.

Clank, H. H. The language-as-fixed-effect fallacy: A critique of language statistics in psychological research. Journal of Verbal Learning and Verbal Behavior, 1973, 12, 335-359.

Clark, H. H., \& Begun, J. S. The semantics of sentence subjects. Language and Speech, 1971, 14, 34-46.

Craik, F. I. M., \& Tulving, E. Depth of processing and the retention of words in episodic memory. Journal of Experimental Psychology: General, 1975, 104, 268-294.
Fillmore, C. J. The case for case. In E. Bach \& R. T. Harms (Eds.), Universals in linguistic theory. New York: Holt, Rinehart, \& Winston, 1968.

Fodor, J. D., Fodor, J. A., \& Garrett, M. F. The psychological unreality of semantic representations. Linguistic Inquiry, 1975, 6, 515-531.

HARRIs, R. J. Memory and comprehension of implications and inferences of complex sentences. Journal of Verbal Learning and Verbal Behavior, 1974, 13, 626-637.

HYde, T. S., \& JENkins, J. J. Differential effects of incidental tasks on the organization of recall of a list of highly associated words. Journal of Experimental Psychology, 1969, 82, 472-481.

Johnson, M. K., Bransford, J. D., \& Solomon, S. K. Memory for tacit implications of sentences. Journal of Experimental Psychology, 1973, 98, 203-205.

Johnson-Laird, P. N., Gibbs, G., \& De Mowbray, J. Meaning, amount of processing, and memory for words. Memory \& Cognition, 1978, 6, 372-375.

KATZ, J. J. Semantic theory. New York: Harper \& Row, 1972.

Kintsch, W. Notes on the structure of semantic memory. In E. Tulving \& W. Donaldson (Eds.), Organization of memory. New York: Academic Press, 1972.

Marslen-Wilson, W., \& Trlen, L. K. Memory and levels of processing in a psycholinguistic context. Journal of Experimental Psychology: Human Learning and Memory, 1976, 2, 112-119.

MCCAWLEY, J. D. Kac and Shibitani on the grammar of killing. In J. Kimball (Ed.), Syntax and semantics (Vol. 1). New York: Academic Press, 1972.

Miluer, G. A., \& Johnson-Laird, P. N. Language and Perception. Cambridge, England: Cambridge University Press, 1976.

Monaco, G. E., \& Harris, R. J. Theoretical issues in the psychology of implication; A reply to Keenan. Journal of Experimental Psychology: General, 1978, 107, 28-31.

Nelson, T. O. Repetition and depth of processing. Journal of Verbal Learning and Verbal Behavior, 1977, 16, 151-171.

Postman, L., \& Kruesi, E. The influence of orienting tasks on the encoding and recall of words. Journal of Verbal Learning and Verbal Behavior, 1977, 16, 353-369.

Shibitani, M. (Ed.) Syntax and semantics (Vol. 6). The grammar of causative constructions. New York: Academic Press, 1976.

Stein, B. S., \& Bransford, J. D. Constraints on effective elaboration: effects of precision and subject generation. Journal of Verbal Learning and Verbal Behavior, 1979, 18, 769-777.

Stein, B. S., Morris, C. D., \& Bransford, J. D. Constraints on effective elaboration. Journal of Verbal Learning and Verbal Behavior, 1978, 17, 707-714.

Thorndike, E. L., \& Lorge, I. The teacher's word book of 30,000 words. New York: Teachers College, Columbia University Press, 1944.

WALSH, D. A., \& Jenkins, J. J. Effects of orienting tasks on free recall in incidental learning: "Difficulty," "effort," and "process" explanations. Journal of Verbal Learning and Verbal Behavior, 1973, 12, 481-488.

\begin{tabular}{llll}
\multicolumn{3}{c}{ Appendix } \\
\hline $\begin{array}{l}\text { Verbs Used in } \\
\text { Experiments } 1 \text { and } 2\end{array}$ & \multicolumn{2}{c}{$\begin{array}{c}\text { Verbs Used in } \\
\text { Experiments } 3 \text { and } 4\end{array}$} \\
\hline blow up & melt & circulate & lengthen \\
burn & open & close & melt \\
end & start & continue & shatter \\
enlarge & stop & decrease & start \\
grow & tighten & develop & stop \\
heal & widen & end & tighten \\
improve & withdraw & enlarge & widen \\
\hline
\end{tabular}

(Received for publication May 27, 1980; revision accepted October 27,1980 .) 